\title{
Les traitements biologiques pour la production d'eau potable
}

\author{
par A. Montiel, B. Welté \\ SAGEP Eau de Paris
}

Si on effectue un historique de l'évolution des traitements des eaux, nous pouvons les classer en deux catégories:

- d'une part, l'évolution des chaînes de potabilisation en fonction des connaissances acquises, notamment au niveau de l'identification de certains composés ou de l'évolution de la législation ou de la demande des consommateurs,

- d'autre part, l'apparition de polluants qui nécessitent des éliminations spécifiques.

\section{I ÉVOLUTION DES CHAÎNES DE POTA- BILISATION D'EAU DE SURFACE}

Lorsque les besoins sans cesse croissants de la quantité d'eau destinée à l'alimentation humaine ont dépassé les possibilités d'utiliser les eaux souterraines ne nécessitant pas de traitement, les distributeurs d'eau se sont orientés vers des eaux de surface rendues potables par traitement.

Les premiers traitements ont simplement consisté à reproduire ce qui se passait dans la nature et la filtration lente est née. Ce traitement ne nécessitant aucun ajout de produit chimique utilisait, d'une part l'effet physique de tamisage obtenu par passage de façon très lente (vitesse de filtration de 2 à $5 \mathrm{~m} / \mathrm{j}$ ) au travers de filtres à sable, d'autre part, un effet biologique à la surface des filtres. Les limites de ce mode de traitement ont très vite été mises en évidence : nécessité de disposer d'une très grande surface de filtration, limite pour les eaux brutes ayant une turbidité trop importante (10 NTU) et très grande sensibilité aux toxiques pouvant être déversés dans les eaux de surface.

Dans un premier temps, des étapes de prétraitement ne nécessitant aucun apport de produits chimiques ont été installées (préfiltration). Ce prétraitement ne permettait pas de régler tous les problèmes cités plus haut. Seule la possibilité de traiter des eaux de 30 NTU de turbidité a été obtenue.

Dans un deuxième temps, la mise au point des traitements physico-chimiques a conduit à la construction d'usines très compactes où tous les problèmes étaient résolus par traitement chimique :

- élimination de la turbidité par coagulation floculation chimique,

- élimination des mieropolluants minéraux par coprécipitation avec, éventuellement, oxydation ou réduction chimique préalable,
- élimination de l'azote réduit (ammonium) par oxydation au chlore (traitement au break point),

- élimination de micropolluants organiques par oxydation chimique (chloration, ozonation).

Cet engouement pour ces traitements chimiques a duré jusque dans les années 1970. A cette époque, certains chercheurs pensaient que toutes les eaux, quel que soit leur degré de pollution, pouvaient être rendues potables par traitement chimique.

Avec les progrès spectaculaires obtenus en analyse des traces de micropolluants dans les eaux, on a mis en évidence la présence de composés organiques induits par l'utilisation de certains traitements chimiques. C'est le cas de la chloration qui peut conduire à la formation de trihalométhanes ou d'autres composés organohalogénés volatils ou non dont la toxicité peut être importante.

Cette découverte a permis une prise de conscience des traiteurs d'eau et la remise en cause du principe : tout traitement chimique.

Comme la chloration de l'eau utilisée pour l'élimination de l'ammoniaque a été la première étape chimique mise en cause, certaines usines ont commencé peu à peu à remplacer cette étape par une étape de nitrification biologique.

Avec l'élimination de la préchloration au break point, est apparu l'effet indirect sur les goûts et odeurs de l'eau, ce qui a induit de nombreuses études sur d'une part, l'élimination par voie biologique de certaines molécules sapides présentes dans l'eau brute, d'autre part la formation de molécules sapides après chloration.

Une deuxième date est à prendre en compte pour la révolution technologique que nous connaissons aujourd'hui dans le traitement des eaux. En effet, dès 1980, la directive Eau potable édictée par la Communauté Economique Européenne précise que les normes définies ne s'appliquent pas qu'à la sortie des usines de potabilisation d'eau, mais aussi à l'arrivée de l'eau chez le consommateur. Cette petite différence a révolutionné toute la philosophie du traitement. En effet, il a été indispensable de prendre en compte les modifications de la qualité de l'eau dans le réseau de distribution et notamment les post-proliférations bactériennes.

Les principaux facteurs influençant les post-proliférations bactériennes dans le réseau (biofilms) sont au nombre de trois :

- Présence d'un bactériostatique en concentration suffisante. 
- Présence de corrosion et des produits induits par la corrosion.

- Présence de nutrients permettant la prolifération de bactéries.

En ce qui concerne la présence de bactériostatiques, la mise en évidence de réactions secondaires et la formation de composés organiques, THM, composés organochlorés non volatils, incite à réduire la concentration en oxydant résiduel dans le réseau de distribution. Ce paramètre ne pouvant être utilisé, les traiteurs d'eau ont effectué des recherches sur les deux autres facteurs: d'une part, l'envoi dans le réseau de distribution d'eaux légèrement incrustantes par rapport à l'équilibre calcocarbonique ou augmentation de la minéralisation pour les eaux très peu minéralisées, d'autre part la réduction des nutrients présents dans l'eau.

Ce dernier facteur a induit des recherches qui ont abouti à la mise au point de méthodes analytiques permettant de mesurer la fraction bioassimilable du carbone organique dissous. Cette mise au point a permis de mieux connaître l'efficacité des chaînes de traitement et par là même de montrer encore l'induction de certaines réactions secondaires, notamment avec l'ozone qui augmente la biodégradation de certaines molécules. Ces découvertes ont donc conduit à l'utilisation d'étapes de traitement permettant l'épuisement du carbone organique assimilable contenu dans les eaux. La filtration finale sur charbon actif en grains a permis d'obtenir ce résultat. Là encore, l'incidence sur les goûts de l'eau n'a pas été négligeable et a conduit globalement à une meilleure qualité de l'eau.

\section{II ÉLIMINATIONS SPÉCIFIQUES DE CERTAINS COMPOSÉS}

Les traitements biologiques peuvent être utilisés pour l'élimination spécifique de certains composés présents dans les eaux. C'est, d'une part, le traitement de certains composés azotés :

- soit l'oxydation biologique de l'azote ammoniacal en azote nitrique (nitrification),

- soit la réduction biologique de l'azote nitrique en azote (dénitrification).

C'est aussi l'oxydation de certains métaux de transition : - oxydation du fer divalent en fer trivalent (déferrisation biologique),

- oxydation du manganèse divalent en manganèse tétravalent (démanganisation biologique).

\section{BUT DES TRAITEMENTS BIOLO- GIQUES}

\subsection{Stabilité biologique de l'eau}

Avec la prise en compte de la dégradation de la qualité de l'eau dans le réseau de distribution, de nombreuses études ont été effectuées sur le biofilm.

Ces études ont permis de montrer qu'en présence d'un biofilm important, la présence d'un bactériostatique dans l'eau distribuée (chlore), même en concentration élevée, ne garantissait pas contre la présence dans l'eau de certains micro-organismes. En effet, le biofilm leur assure un effet protecteur vis-à-vis des bactériostatiques.
L'idée d'avoir pour garantie un résiduel de chlore important est peu à peu appelée à disparaître. Certains chercheurs proposent même l'eau sans chlore en distribution. Certaines villes comme Amsterdam ne distribuent-elles pas de l'eau sans chlore, sans bactériostatique.

En effet, le seul moyen de réduire ce biofilm est de réduire au maximum la présence dans l'eau de carbone organique bioassimilable soluble (BDOC) et la présence de substance pouvant introduire la protection des micro-organismes : c'est le cas des colloïdes.

Des consignes de qualité sont donc en cours d'élaboration sur le BDOC mais aussi sur la turbidité résiduelle et les valeurs inférieures à 1 NTU sont avancées. Certains chercheurs proposent de 0,1 à 0,3 NTU. En effet, la turbidité joue aussi un rôle très important sur l'élimination des microorganismes.

Une élimination très poussée de la turbidité permet une très bonne rétention de micro-organismes très résistants au chlore : giardia, cryptosporidium, amibes et une réduction de 2 à $3 \log$ du nombre de bactéries.

Une élimination très poussée de la turbidité empêche l'effet protecteur des colloïdes vis-à-vis du chlore. Cette élimination évite aussi certaines postfloculations dans le réseau de distribution.

\subsection{Elimination de l'ammonium}

La présence dans l'eau traitée d'ammonium conduit dans le réseau de distribution à une oxydation biologique qui induit d'une part, des proliférations bactériennes, mais aussi la présence de nitrites. Cet élément doit donc être absent des eaux distribuées.

\subsection{Elimination des nitrates et nitrites}

La présence dans l'eau de fortes teneurs en nitrates (supérieures à $50 \mathrm{mg} / \mathrm{l}$ ) ou nitrites (supérieures à $0,1 \mathrm{mg} / \mathrm{l}$ ) conduit à considérer que l'eau ne peut plus être utilisée par toute la population. La plupart des législations existantes s'accordent à dire que les eaux dont la teneur en nitrates est supérieure à $50 \mathrm{mg} / \mathrm{l}\left(\mathrm{NO}_{3}\right)$ ne peuvent être utilisées pour l'alimentation des nourrissons et des femmes enceintes.

L'élimination des nitrates peut être obtenue par deux moyens :

- soit une dénitratation par échange ionique,

- soit une dénitrification biologique.

\section{IV 畨 CONCLUSION}

Les techniques utilisées pour la potabilisation des eaux sont en constante évolution. Ces évolutions sont fonction des progrès faits d'une part, en épidémiologie et toxicologie qui permettent d'apprécier le risque induit par la présence dans l'eau d'un composé et d'autre part, par l'évolution des techniques analytiques.

Le tout chimique utilisé au début des traitements de potabilisation a été remplacé par le tout biologique. On assiste aujourd'hui à un équilibre chimique-biologique et aussi à l'apparition de techniques purement physiques (membranaires). L'avenir conduira, à n'en pas douter, à un équilibre physique, chimique et biologique. 